\title{
Normal persistent currents
}

\author{
By Ulrich Eckern and Peter Schwab \\ Institut für Physik, Universität Augsburg, D-86135 Augsburg, Germany
}

\begin{abstract}
This article reviews recent advances in mesoscopic physics, namely experimental and theoretical investigations of persistent (equilibrium) currents in normal metal rings. While theory and experiment are consistent in the ballistic regime, theoretical studies so far have not been able to account for the unexpectedly huge currents observed experimentally in disordered (diffusive) samples, in which the elastic mean free path is much less than the circumference of the rings.
\end{abstract}

\section{Contents}

PAGE

1. Introduction

1.1. General theory

1.2. Correlation functions 388

1.3. Experimental results 390

2. Non-interacting electrons 390

2.1. One dimension 390

2.2. Average current 393

2.3. Correlations 395

3. Interacting electrons 397

3.1. Average current 397

3.2. Correlations 399

4. Further developments 400

4.1. Spin and magnetic field effects $\quad 400$

4.2. Higher correlation functions 401

4.3. Capacitance models $\quad 402$

4.4. Superconducting rings 402

Acknowledgements $\quad 403$

References 403

\section{Introduction}

The question of the magnetic properties of ring molecules and metallic rings has a long history [1-5]. In analogy to the Aharonov-Bohm effect, that is the tuning of the interference pattern in a double-slit experiment through the enclosed flux, it can be expected that the magnetization, or the corresponding 'persistent current', has a flux periodic contribution. In this sense, and for sufficiently low temperatures, metallic rings are very similar to ring molecules. But how large is the effect for a mesoscopic disordered ring? Is it experimentally observable with today's technology?

Three successful experimental investigations, which showed that persistent currents exist in mesoscopic metal $[6,7]$ and semiconductor $[8(a)]$ rings (a more recent experiment is described in $[8(b)])$, were most important for the recent advancement in this field. In reaction to these experiments, the phenomenon of persistent currents 
has been discussed theoretically in various papers [9-28]. Selected aspects of the theory are presented in this article and compared with the experimental findings.

\subsection{General theory}

In the following we consider, for simplicity, an idealized situation where the width of the metal ring is so small (compared with the circumference $L$ ) that magnetic field penetration into the metal can be neglected. Without field penetration, the energy and the thermodynamic potential $K(\phi)$ of a given sample depend on the magnetic flux $\phi$ (and not explicitly on the magnetic field). As the persistent current $I(\phi)$ is an equilibrium phenomenon $\dagger$, it can be calculated by taking the derivative according to

$$
I(\phi)=-\frac{\partial K(\phi)}{\partial \phi}
$$

Depending on the variables to be kept constant in the experiment $K(\phi)$ is given by $F(N, \phi)$ or $\Omega(\mu, \phi)$ for the canonical and the grand ensemble, respectively.

In the case of time-reversal symmetry, $K(\phi)$ is an even function of $\phi$ and, in the idealized situation, it is also periodic in $\phi$ with primitive period $\phi_{0}=h / e \equiv 2 \pi \hbar / e$. It therefore follows that

$$
I(-\phi)=-I(\phi), \quad I\left(\phi+\phi_{0}\right)=I(\phi),
$$

for any realization of the impurity positions, and that the following Fourier decomposition is valid:

$$
\begin{gathered}
K(\phi)=\sum_{m=0}^{\infty} K_{m} \cos \left(\frac{2 \pi m \phi}{\phi_{0}}\right) \\
I(\phi)=\sum_{m=1}^{\infty} I_{m} \sin \left(\frac{2 \pi m \phi}{\phi_{0}}\right) .
\end{gathered}
$$

Clearly, the following relation holds $(m>0)$ :

$$
I_{m}=\frac{e m K_{m}}{\hbar} .
$$

For completeness, we would mention that the persistent current generates a magnetic moment $M(\phi)=\pi(L / 2 \pi)^{2} I(\phi)$, directed perpendicular to the ring.

\subsection{Correlation functions}

The properties of mesoscopic samples are very sensitive to the actual impurity configuration. It is therefore appropriate to introduce an ensemble of mesoscopic samples which have been fabricated to the same specifications but differ in the positions of their defects. Hence the persistent currents $I(\phi)$, that is the set $\left\{I_{m}\right\}$ of its Fourier amplitudes, should be considered as stochastic quantities which are completely characterized if all correlation functions are known. Thus, we have to consider the set of all $k$-point correlation function

$$
\begin{aligned}
& \mathscr{M}_{I}\left(\phi_{1}, \ldots, \phi_{k}\right)=\left\langle I\left(\phi_{1}\right) I\left(\phi_{2}\right) \cdots I\left(\phi_{k}\right)\right\rangle_{\mathrm{c}}, \\
& \mathscr{M}_{I}\left(m_{1}, \ldots, m_{k}\right)=\left\langle I_{m_{1}} I_{m_{2}} \cdots I_{m_{\mathrm{k}}}\right\rangle_{c},
\end{aligned}
$$

$\uparrow$ The transport properties of mesoscopic rings in a magnetic field have been reviewed, for example by Washburn and Webb [29]. 
where the angular brackets $\langle\cdots\rangle$ mean the average with respect to the impurity ensemble introduced above. Specifically, $\langle\cdots\rangle_{\mathrm{c}}$ means the cumulant correlation functions. For instance,

$$
\left\langle I\left(\phi_{1}\right) I\left(\phi_{2}\right)\right\rangle_{\mathrm{e}}=\left\langle I\left(\phi_{1}\right) I\left(\phi_{2}\right)\right\rangle-\left\langle I\left(\phi_{1}\right)\right\rangle\left\langle\left(\phi_{2}\right)\right\rangle .
$$

In a graph theory, the cumulants are defined as the set of connected diagrams.

As a rule, it is more convenient to calculate the correlation function of thermodynamic potentials and to calculate the currents by taking the derivative with respect to the fluxes as shown in equation (1). There are arguments, which show that the stochastic properties of the persistent currents are adequately obtained within the grand canonical ensemble. Thus we introduce the following set of $k$-point correlation functions:

$$
\mathscr{H}_{\Omega}\left(\phi_{1}, \ldots, \phi_{k}\right)=\left\langle\Omega\left(\phi_{1}\right) \Omega\left(\phi_{2}\right) \cdots \Omega\left(\phi_{k}\right)\right\rangle_{\mathrm{e}} .
$$

It is obvious that the magnitude of the persistent currents may equally be characterized by energies. In this respect, one should recognize that a mesoscopic metal ring displays a variety of quite different energy scales as follows:

(1) Fermi energy $\epsilon_{\mathrm{F}}$;

(2) elastic scattering rate $h / \tau$;

(3) energy $\hbar v_{\mathrm{F}} / L$ to localize an electron in one half of the ring;

(4) Thouless energy $E_{\mathrm{c}}=h D / L^{2}$;

(5) mean level spacing $\Delta=1 / 2 \mathscr{N}^{0} \mathscr{V} \propto \epsilon_{\mathrm{F}} / N$.

Here $\epsilon_{\mathrm{F}}$ and $v_{\mathrm{F}}\left(=p_{\mathrm{F}} / m\right)$ are the energy and velocity of electrons at the Fermi level, and $\mathscr{N}^{0}=m p_{\mathrm{F}} / 2 \pi^{2} \hbar^{3}$ is the density of states. The diffusion constant is $D=v_{\mathbf{F}} l / 3$, where $l=v_{\mathbf{F}} \tau$ is the mean free path. Furthermore, $L$ is the circumference, $\mathscr{N}=\mathscr{A} L$ is the volume of the mesoscopic ring and $N \propto \mathscr{F} p_{\mathrm{F}}^{3} / h^{3}$ is the total number of electrons.

Of the hierarchy of correlation functions, the average current $\langle I(\phi)\rangle$ and the two-point correlator $\left\langle I(\phi) I\left(\phi^{\prime}\right)\right\rangle_{\mathrm{c}}$ have been calculated by several workers. There seems to be general agreement on the following properties:

$$
\left\langle I_{2 p+1}\right\rangle=0 ; \quad\left\langle I_{m} I_{m^{\prime}}\right\rangle_{\mathrm{c}}=0, \quad m \neq m^{\prime} .
$$

For a general orientation, we list some results, by order of magnitude and for $T \approx 0$.

(1) Non-interacting electrons, ballistic limit $(l \gg L)$, one channel: $I_{m} \propto e v_{\mathrm{F}} / L$; the sign depends on the number of electrons.

(2) Non-interacting electrons, diffusive limit $(l \ll L):\left\langle I_{2 p}\right\rangle \propto 0$ (grand canonical ensemble) or $\left\langle I_{2 p}\right\rangle \propto e \Delta / \hbar$ (canonical ensemble), and $\left\langle I_{m}^{2}\right\rangle_{\mathrm{c}} \propto\left(e E_{\mathrm{c}} / \hbar\right)^{2}$.

(3) Interacting electrons, diffusive limit: $\left\langle I_{2 p}\right\rangle \propto e E_{c} / h$; however, the issue of correlations is still open.

Theoretical models on persistent currents differ according to whether or not the Coulomb interaction is taken into account. With non-interacting fermions, the problem is easily solved in a perfect ring geometry [9]. Inclusion of impurity scattering already leads to non-trivial complications. One may resort to computer calculations $[12,28]$, to random matrix theory, to the diffusion-cooperon expansion [30] within the impurity technique for Green functions [31], or to the supersymmetry technique [32]. For a new approach to the interacting electron problem with disorder, see also [26]. 


\subsection{Experimental results}

The experimental observations reported so far contain different information. In the experiment by the Bell group [6], the persistent current of an ensemble of about $10^{7}$ copper rings has been measured; this supplies information on the average current $\left\langle I_{m}\right\rangle$. The odd harmonics seem to be absent; the average value of the first non-vanishing harmonic $\left\langle I_{2}\right\rangle$ is found to be approximately $0.6 e E_{\mathrm{c}} / \mathrm{h}$. Other relevant parameters are as follows: $L \approx 2 \times 10^{-4} \mathrm{~cm}, l / L \approx 1 / 70, \Delta \approx 0.2 \mathrm{mK}, E_{\mathrm{c}} \approx 25 \mathrm{mK}$ and $\hbar v_{\mathrm{F}} / L \approx 5 \mathrm{~K}$. (For convenience, energies are here given in temperature units.) This agrees roughly with the theory for interacting electrons in the diffusive limit (see, however, 3.1). In the experiments by the IBM group [7], the persistent currents of three different single gold rings have been measured. In such a single-ring experiment, one expects the observed current to be of the order $\left\langle I_{m}^{2}\right\rangle_{c}^{1 / 2}$ (usually this is very much larger than the average current $\left.\left\langle I_{m}\right\rangle\right)$. It was found that $I_{1} \sim(0.3-2 \cdot 0) e v_{\mathrm{F}} / L$. The other relevant parameters are as follows: $L \approx 8 \times 10^{-4} \mathrm{~cm}, l / L \approx 1 / 110, \Delta \approx$ $0.02 \mathrm{mK}, E_{\mathrm{c}} \approx 4 \mathrm{mK}$ and $\hbar v_{\mathrm{F}} / L \approx 1 \mathrm{~K}$.

Note that the relation $\left\langle I_{1}^{2}\right\rangle_{\mathrm{c}} \gg\left\langle I_{1}\right\rangle^{2}$ requires the sign of the observed currents to vary randomly from sample to sample. Experimentally, the sign of the current has been positive for the two samples that have been checked, but obviously no conclusion can be drawn from such a limited sampling.

These two experiments are clearly in the diffusive limit. In contrast, for the semiconductor ring studied in $[8(a)]$, the mean free path is comparable with the circumference of the ring. For such a situation, the result $I_{1} \propto e v_{F} / L$ is not so surprising.

\section{Non-interacting electrons}

2.1. One dimension

As the simplest example, consider non-interacting electrons on a one-dimensional ring without disorder. We then have to solve the Schrödinger equation

$$
\frac{\hat{p}^{2}}{2 m} \psi(x)=\epsilon \psi(x)
$$

with periodic boundary conditions. The solutions are plane waves, with the wavevector $k=p / h$ given by

$$
k_{n}=\frac{2 \pi}{L} n
$$

where $n$ is an integer. Applying a constant magnetic field $B$, perpendicular to the ring, equation (11) is modified such that $\hat{p} \rightarrow \hat{p}+e A$, with $A=B r / 2=B L / 4 \pi$. On the other hand, the magnetic flux $\phi$ through the ring equals $B L^{2} / 4 \pi$; so we obtain

$$
k_{n}=\frac{2 \pi}{L} n+\frac{e}{\hbar} A=\frac{2 \pi}{L}\left(n+\frac{\phi}{\phi_{0}}\right),
$$

that is the energy levels $\epsilon_{n}=\hbar^{2} k_{n}^{2} / 2 m$ are shifted by the magnetic flux. Clearly, the spectrum $\left\{\epsilon_{n}\right\}$ is periodic in $\phi$ with period $\phi_{0}$; so we may restrict ourselves to the interval from $-\phi_{0} / 2$ to $\phi_{0} / 2$ and plot the energy levels against flux (figure 1 ). In the next step, it is straightforward to determine the current carried by the $n$th level: 


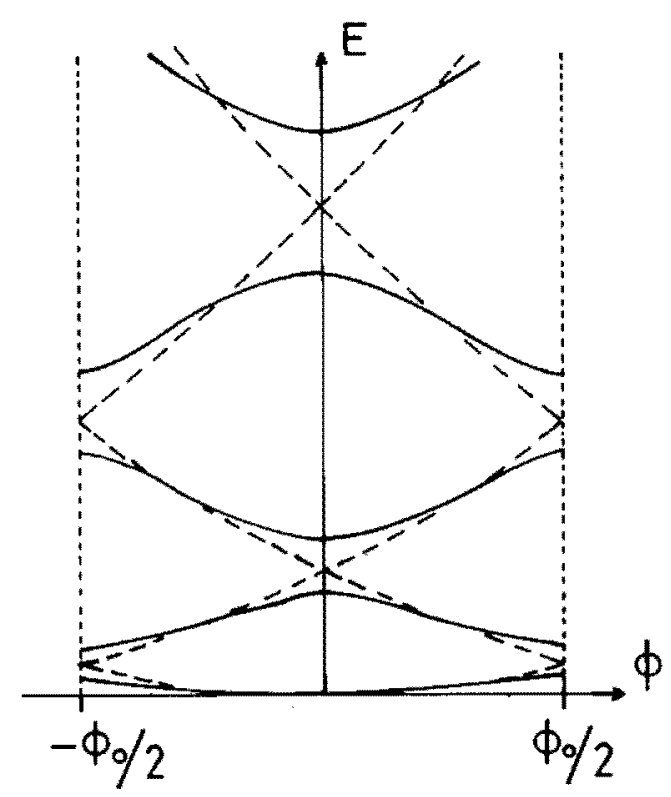

Figure 1. Low-lying energy levels of a one-dimensional non-interacting electron gas on a ring against magnetic flux in the clean case (- - ) and in the presence of weak disorder (- In the clean limit, it is apparent that the contributions from successive levels to the current cancel near $\phi=0, \pm \phi_{0} / 2$. The corresponding jumps in the total current are washed out by disorder.

$I_{n}=-\partial \epsilon_{n} / \partial \phi$. The total persistent current is then given by

$$
I=\sum_{n} I_{n}
$$

where the summation is over the $N$ lowest (occupied) levels (we consider $T=0$ and ignore the spin in this section). For the following discussion, we label the states differently, such that $\epsilon^{1}<\epsilon^{2}<\epsilon^{3}<\cdots$. As an example, consider a small positive flux $\phi \rightarrow+0$. Then $\epsilon^{1}$ carries no current, $\epsilon^{2}$ a positive current, $\epsilon^{3}$ a negative current, and so on (see figure 1, broken curves). However, it is evident that the contributions from the second and the third level and so on cancel, which implies that the total current is determined by the last electron of highest energy, that is $I \propto e v_{\mathrm{F}} / L$ or $I \approx 0$ for $N$ even or odd respectively! To be more precise, the result for odd $N$ is given by

$$
I(\phi)=-\frac{e v_{\mathrm{F}}}{L} \frac{2 \phi}{\phi_{0}}
$$

in the considered interval (and periodically continued), while the result for even $N$ is just shifted by half a flux quantum.

Using the analogy to band-structure calculations in solid-state physics, it is possible to obtain a rough idea of what happens in the presence of weak disorder on the ring. In solid-state physics, one starts with the parabolic dispersion and observes that, for a weak lattice potential, the dispersion is deformed near the boundaries of the Brillouin zone, with an energy gap opening. A similar result is expected here, as indicated in figure 1 , which leads to a smearing of the discontinuities discussed above.

For illustration, we may also consider a delta-like impurity in our one-dimensional model, adding the potential $V(x)=a \delta\left(x-x_{0}\right)$ to the Hamiltonian. (The point $x_{0}$ 
is arbitrary, and we choose $x_{0}=0$.) A standard calculation of quantum mechanics determines the transfer matrix $\mathscr{T}$ of this potential, which is a $2 \times 2$ matrix connecting the amplitudes of the plane waves on the left and the right side of the impurity. The result is

$$
\mathscr{T}=\left[\begin{array}{cc}
\left(1-\frac{\mathrm{i} m a}{\hbar^{2} k}\right) & -\frac{\mathrm{i} m a}{\hbar^{2} k} \\
+\frac{\mathrm{i} m a}{\hbar^{2} k} & \left(1+\frac{\mathrm{i} m a}{\hbar^{2} k}\right)
\end{array}\right] .
$$

Clearly, $\mathscr{T}_{11}=\mathscr{T}_{22}^{*}, \mathscr{T}_{12}=\mathscr{T}_{21}^{*}$, and $\operatorname{det} \mathscr{T}=1$ (which follows from time-reversal symmetry and probability conservation). Here we use a gauge in which the vector potential does not appear in the Hamiltonian but rather in the boundary condition, $\psi(x+L)=\exp (\mathrm{i} \varphi) \psi(x)$. From the above transfer matrix, one easily finds the condition which determines $k$ and hence the energy spectrum $\hbar^{2} k^{2} / 2 m$ :

$$
\operatorname{Re}\left[\mathscr{T}_{11} \exp (i k L)\right]=\cos \varphi,
$$

where $\varphi=2 \pi \phi / \phi_{0}$ or, more explicitly,

$$
\cos (k L)+\frac{m a \sin (k L)}{\hbar^{2} k}=\cos \varphi
$$

The summation over energy levels can generally be quite difficult. As a simple example, let us consider the case of a strong impurity. In the limit $m a \gg \hbar^{2} k$, we have to a first approximation

$$
k_{n}^{0}=\frac{\pi n}{L}
$$

and the (weak) dependence on the flux can be calculated perturbatively. The flux-dependent correction is

$$
k_{n}^{1}=(-)^{n} \frac{\hbar^{2} k_{n}^{o}}{m a L}\left[\cos \varphi-(-)^{n}\right] .
$$

Thus a strong impurity ( $a \gg \hbar v_{\mathrm{F}}$, where $v_{\mathrm{F}}=\hbar k_{\mathrm{F}} / m$ and $k_{\mathrm{F}} \equiv \pi N / L$ ) lifts the degeneracy apparent in the clean limit at zero flux and at the zone boundaries. We also deduce that the current carried by the $n$th level is reduced in order of magnitude by the approximate factor $\hbar v_{n} / a$ :

$$
I_{n}=-\frac{\hbar^{2} k_{n}^{0}}{m} \frac{\partial k_{n}^{1}}{\partial \phi}=(-)^{n}\left(\frac{h^{2} k_{n}^{0}}{m}\right)^{2} \frac{e / \hbar}{a L} \sin \varphi
$$

In performing the $n$ summation, we encounter the expression

$$
\sum_{n=1}^{N}(-)^{n} n^{2}=\frac{(-)^{N}\left(N^{2}+N\right)}{2}
$$

For a negative potential, $a<0$, there exists a localized state, that is a solution of equation (17) with imaginary $k$ and and energy less than zero. For a strong impurity, the current carried by this state is negligible but, since the localized state is always occupied, for a given number $N$ of electrons, the number of current-carrying states is reduced by one. The final result, including the second-order term, is the following 
$\left(a^{*}=h v_{\mathrm{F}} / \pi\right)$

$$
I(\phi)=\frac{\pi e v_{\mathrm{F}}}{2 L}\left[(-)^{N} \frac{a^{*}}{|a|} \sin \varphi+\left(\frac{a^{*}}{a}\right)^{2} \sin (2 \varphi)+\cdots\right] .
$$

The persistent current is clearly reduced from the clean limit value and is given approximately by a sine function. However, it should be noted that the dominant contribution, proportional to $\sin \varphi$, is very sensitive to an averaging procedure involving different particle numbers. In view of this sensitivity, we may expect that the average persistent current, even in a more realistic situation, is considerably smaller than the current in a single ring (which can be of either sign), and in particular that the dominant flux periodicity of the average signal is $\phi_{0} / 2$, as confirmed experimentally.

The above considerations can be applied equally well to a tight-binding model; in that case the spectrum is $-2 t \cos \left(k a_{0}\right)$, where $a_{0}$ is the lattice constant.

\subsection{Average current}

We now consider a more realistic situation, in which the transverse is finite so that the number of transverse channels is large, $\mathscr{A} k_{\mathrm{F}}^{2} \gg 1$, and also include disorder with an elastic mean free path $l$ such that $1 / k_{\mathrm{F}} \ll l \ll L$. Hence the electron motion is diffusive, and it is necessary to use the impurity technique for the Green functions and the cooperon-diffuson expansion [30]. A detailed presentation of all intermediate steps is not intended here; so we restrict ourselves below, except for the next section, to the essential results.

In order to determine the average persistent current, consider first the grand canonical potential

$$
\Omega(\mu)=-2 \mathscr{F} T \int \mathrm{d} E \mathscr{N}(E) \ln \left[1+\exp \left(-\frac{(E-\mu)}{T}\right)\right]
$$

where the factor two is due to the spin, $\mathscr{V}=\mathscr{A L}$ is the volume, and $\mathscr{N}(E)$ is the density of states. The energy levels and hence the density of states depend on the flux, but it is well known that the average density of states, $\mathscr{N}^{0} \equiv\langle\mathscr{N}(E)\rangle$, is a flux-independent quantity except for corrections which are exponentially small, proportional to $\exp (-L / 2 l)$. Thus the average current, as computed in the grand canonical ensemble, is negligibly small, which is not surprising in view of the sensitivity to changes in the particle number.

Thus we are led to consider a situation where, as a function of the flux, the particle number is kept exactly fixed, that is a canonical ensemble $[16,18]$. Since

$$
N=2 \mathscr{V} \int \mathrm{d} E \mathscr{N}(E) f(E-\mu),
$$

where $f(E-\mu)$ denotes the Fermi function and, since the density of states fluctuates from sample to sample, it is clear that the chemical potential has to acquire a fluctuating contribution, $\mu=\mu^{0}+\mu^{1}$. Working in second order in the fluctuating quantities, the free energy is easily determined. First, define $\mu^{0}$ through the relation

$$
N=2 \mathscr{V} \int \mathrm{d} E \mathscr{N}^{0} f\left(E-\mu^{0}\right)
$$


(Note that the variation in $\mathscr{N}^{0}$ on the scale of the Fermi energy is not of interest here; so it is not explicitly indicated.) Second, we obtain

$$
\mathscr{N}^{0} \mu^{1}=-\int \mathrm{d} E \mathscr{N}^{1} f\left(E-\mu^{0}\right)
$$

In the third step, expand $F(N)=\Omega(\mu)+\mu N$ in second order, $F=F^{0}+\delta F$, with the result

$$
\delta F=-\frac{1}{2}\left(\frac{\partial^{2} \Omega}{\partial \mu^{2}}\right)_{\mu_{0}}\left(\mu^{1}\right)^{2} \approx \mathscr{N}^{0} \mathscr{V}\left(\mu^{1}\right)^{2},
$$

where the flux sensitivity is in the second factor. Defining $\delta N=2 \mathscr{N}^{0} \mathscr{V} \mu^{1}$, we arrive at

$$
\langle\delta F\rangle=\frac{1}{2} \Delta\left\langle(\delta N)^{2}\right\rangle_{\mu},
$$

where $\Delta \equiv\left(2 \mathscr{N}^{0} \mathscr{V}\right)^{-1}$. The subscript $\mu$ indicates that the fluctuations of the particle number in this expression have to be calculated within the grand canonical ensemble. Using equation (27), this correlator is easily related to the fluctuations of the density of states, which were determined in [33]. The relevant Feynman diagram is shown in figure $2(a)$ where, as a characteristic feature, two cooperon ladders are apparent. An alternative approach is presented in the next section. We emphasize that the flux sensitivity is determined by the (singular) low-frequency long-wavelength contribution of the cooperons. The final result is the following:

$$
\langle\delta F\rangle=\frac{2 \Delta}{\pi \hbar} T \sum_{\omega>0} \sum_{\mathbf{q}} \frac{\omega}{\left(\omega+D q^{2}\right)^{2}}
$$

where $\omega=2 \pi T n / h$ are Matsubara frequencies. It is important to realize now that $\mathbf{q}$ is a sum (difference) of two wave-vectors for the cooperon (diffusion). Furthermore, it has to be taken as one dimensional in the above expression. Hence

$$
q \rightarrow \frac{2 \pi}{L}\left(n+\frac{\phi \pm \phi}{\phi_{0}}\right)
$$

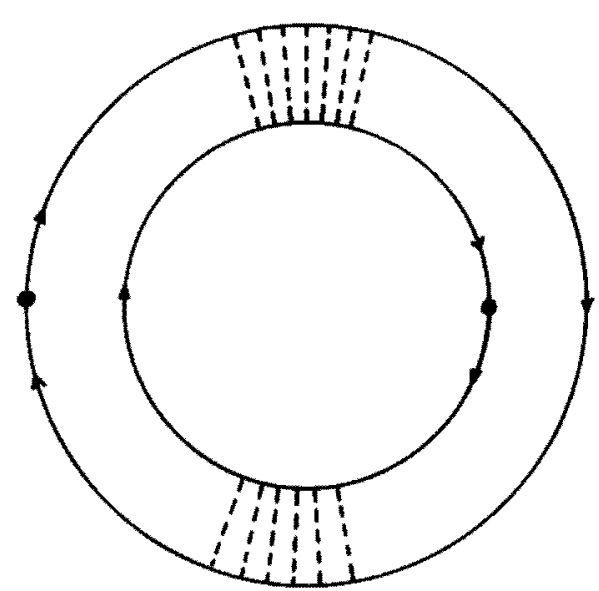

(a)

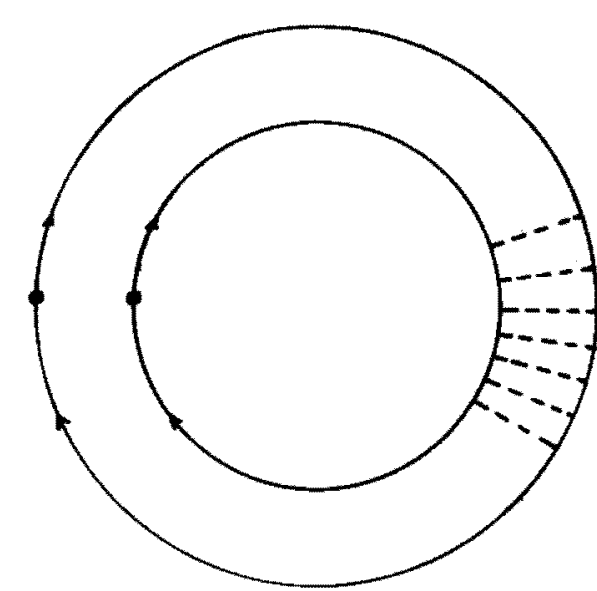

(b)

Figure 2. Relevant diagrams contributing $(a)$ to the flux-sensitive part of the fluctuations of the total particle number (equation (29)) and $(b)$ to the fluctuations of the local density (equation (50)). 
for the cooperon and the diffuson respectively. At present, the diffuson is unimportant (remember that we have to take another derivative with respect to $\phi$ ). Clearly, the dominant flux periodicity is $\phi_{0} / 2$.

As a technical detail, note that, for any periodic function of the type

$$
X(\zeta)=\sum_{n=-\infty}^{+\infty} G\left(\left[D\left(\frac{2 \pi}{L}\right)^{2}(n-\zeta)^{2}\right]\right)=\sum_{m=-\infty}^{+\infty} \exp (2 \pi i m \zeta) X_{m}
$$

the Fourier coefficients $X_{m}$ can be calculated according to

$$
X_{m}=\frac{1}{2 \pi} \int_{-\infty}^{+\infty} \mathrm{d} x \exp (-(\mathrm{i} m x)) G\left(\left[E_{\mathrm{c}} x^{2}\right]\right)
$$

Since $X_{-m}=X_{m}$, only terms $\propto \cos (2 \pi m \zeta)$ survive the summation in equation (32).

In performing this programme, we find the following result for zero temperature:

$$
\left\langle I_{2 p}\right\rangle=\frac{4 e \Delta}{\pi^{2} h},
$$

which in particular is independent of $p$. In performing the $p$ summation, we finally obtain

$$
I(\phi)=\frac{e \Delta}{\pi^{3} \hbar} \cot \left(\frac{2 \pi \phi}{\phi_{0}}\right)
$$

which diverges $\propto \phi^{-1}$ as $\phi \rightarrow 0$. This non-physical result can be cured by introducing a finite phase breaking rate into the denominator of equation (30), $\omega \rightarrow \omega+\gamma$, and it can be argued $[16,18]$ that $\gamma \approx \Delta / h$. As a result, at $\phi \propto\left(\Delta / E_{\mathrm{c}}\right)^{1 / 2} \phi_{0}$, there is a cross-over to a linear flux dependence

$$
I(\phi) \propto \frac{e E_{\mathrm{c}}}{\hbar} \frac{\phi}{\phi_{0}}
$$

for very small fluxes. This behaviour has been confirmed within the supersymmetry method $[19,20]$. In view of the singular flux dependence, the above results only apply in the regime $T<\Delta$, which is not accessible to experiment (at present). For higher temperatures $T \approx E_{\mathrm{c}}$, the higher harmonics are rapidly suppressed, such that a sinusoidal flux dependence remains, with an amplitude given by the average level spacing $\Delta$. The temperature dependence is discussed below in more detail, in comparison with the temperature dependence of the average current induced by the Coulomb interaction.

\subsection{Correlations}

The differences between the canonical and the grand canonical ensemble appear to be negligible in the problem of stochastic fluctuations. Therefore we shall consider the grand canonical ensemble, and we shall calculate the following two-point correlator:

$$
\mathscr{M}_{\Omega}\left(\phi, \phi^{\prime}\right)=\left\langle\Omega(\phi) \Omega\left(\phi^{\prime}\right)\right\rangle_{\mathrm{c}}
$$

In quantum field theory, the grand canonical potential and its correlation functions are represented graphically by vacuum diagrams. Furthermore, within the impurity technique for the Green functions [30], the flux dependence is obtained from the 


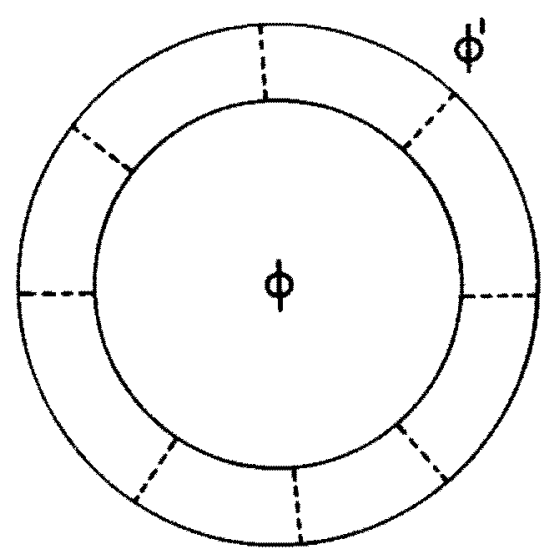

Figure 3. Relevant contribution to the fluctuations of the grand potential (equation (38)). The symmetry of the diagram is apparent.

dominant diffuson and cooperon contributions (particle-hole and particle-particle ladders). Note that diffuson and cooperon contribute to the two-point correlation symmetrically, the flux dependence being $\phi-\phi^{\prime}$ and $\phi+\phi^{\prime}$ respectively; hence, in the diagram for $\mathscr{M}_{\Omega}\left(\phi, \phi^{\prime}\right)$, we omit the arrows on the electronic loops implying that both senses of circulation have to be considered.

The relevant expression for non-interacting electrons is shown in figure 3 where, by symmetry of the graph, the term with $M$ connecting impurity lines has the non-trivial combinatorial factor $M^{-1}$. Hence a typical term is given by $(x \equiv(\mathbf{r}, \tau)$ comprises space and time)

$$
\begin{array}{r}
\frac{1}{M}\left(2 \pi \mathscr{N}^{0}\right)^{-M} \int \mathrm{d} x_{1} \cdots \mathrm{d} x_{M} \Gamma\left(x_{1}-x_{1}^{\prime}\right) \mathscr{G}\left(x_{1}, x_{2}\right) \mathscr{G}\left(x_{1}^{\prime}, x_{2}^{\prime}\right) \\
\cdots \Gamma\left(x_{M}-x_{M}^{\prime}\right) \mathscr{G}\left(x_{M}, x_{1}\right) \mathscr{G}\left(x_{M}^{\prime}, x_{1}^{\prime}\right)
\end{array}
$$

where we have associated $\Gamma\left(x_{i}-x_{i}^{\prime}\right) / 2 \pi \mathscr{N}^{0}$ with each impurity line connecting $\Omega(\phi)$ with $\Omega\left(\phi^{\prime}\right)$, represented in figure 3 by the two electronic loops. We note that the series starts with $M=2$; the term with $M=1$ has to be omitted for reasons of charge neutrality.

We now consider the above expression in momentum space. Here we encounter the expression

$$
I_{0}(\mathbf{q}, \omega)=\frac{\Gamma}{2 \pi \mathcal{N}^{0}} \int \frac{\mathrm{d}^{3} k}{(2 \pi)^{3}} \mathscr{G}(\mathbf{k}, \epsilon) \mathscr{G}(\mathbf{k}-\mathbf{q}, \epsilon-\omega) .
$$

In the standard approximation valid for $|\omega|, v_{\mathrm{F}} q \ll \epsilon_{\mathrm{F}}$ and in the frequency regime $\epsilon(\epsilon-\omega)<0$, it is given by

$$
I_{0}(\mathbf{q}, \omega)=\frac{\Gamma}{v_{\mathrm{F}} q} \tan ^{-1}\left(\frac{v_{\mathrm{F}} q}{|\omega|+\Gamma}\right)
$$

Hence

$$
\mathscr{M}_{\Omega}=4 T \sum_{\omega, q} \sum_{M=2}^{\infty} \frac{1}{M}\left[I_{0}(\mathbf{q}, \omega)\right]^{M},
$$

where a spin factor of four has been included. From this expression, the dominant flux dependence is extracted by considering the contribution from small $\omega$ and $\mathbf{q}$, 
that is $|\omega| \tau<1$ and $q l<1$, by noting that in this regime

$$
1-I_{0} \approx \tau\left(|\omega|+D q^{2}\right)
$$

where $1 / \tau=\Gamma$. In performing the $M$ summation in equation (41), we arrive at

$$
\mathscr{A}_{\Omega}=\frac{4 T}{\pi} \sum_{\omega>0} \sum_{\mathbf{q}} \omega\left[\ln \left(\frac{1 / \tau}{\omega+D q^{2}}\right)-I_{0}\right] .
$$

Again, for the ring geometry, $\mathbf{q}$ has to be taken to be one dimensional so that

$$
\mathbf{q} \rightarrow \frac{2 \pi}{L}\left(n-\frac{\phi \pm \phi^{\prime}}{\phi_{0}}\right)
$$

with the upper and lower signs for the cooperon and diffuson respectively.

Note that, in the Fourier representation, the combined contribution of cooperon and diffusion,

$$
\cos \left(2 \pi m \frac{\phi+\phi_{0}}{\phi_{0}}\right)+\cos \left(2 \pi m \frac{\phi-\phi^{\prime}}{\phi_{0}}\right)=2 \cos \left(2 \pi m \frac{\phi}{\phi_{0}}\right) \cos \left(2 \pi m \frac{\phi^{\prime}}{\phi_{0}}\right)
$$

leads to a result where the thermodynamic potentials are even functions of the fluxes, as it should be. Eventually, we obtain

$$
\left\langle I_{m} I_{m^{\prime}}\right\rangle_{\mathrm{e}}=\delta_{m, m^{\prime}} \frac{96}{\pi^{2}}\left(\frac{e E_{\mathrm{c}}}{\hbar}\right)^{2} \frac{1}{m^{3}},
$$

which has already been outlined in section 2 .

As mentioned in the previous section, the above analysis can easily be extended to determine the fluctuations of the particle number. Hence let us consider a case where the chemical potentials on the two rings differ; we denote them by $\mu$ and $\mu^{\prime}$. Then, with the replacement

$$
\omega+D q^{2} \rightarrow \omega+D q^{2}+\mathrm{i}\left(\mu-\mu^{\prime}\right)
$$

in equation (43), and taking the real part of the resulting expression, we obtain the correlation function

$$
\left\langle\Omega(\mu, \phi) \Omega\left(\mu^{\prime}, \phi^{\prime}\right)\right\rangle_{\mathrm{c}}
$$

This quantity is differentiated with respect to $\mu$ and $\mu^{\prime}$, yielding the quantity

$$
\left\langle N(\mu, \phi) N\left(\mu^{\prime}, \phi^{\prime}\right)\right\rangle_{\mathrm{c}} \text {. }
$$

Finally, setting $\mu=\mu^{\prime}$ and $\phi=\phi^{\prime}$, we recover precisely the expression required to determine the correction to the free energy as given in equation (29). Note that, for equal fluxes, only the cooperon gives a relevant contribution to the average persistent current.

\section{Interacting electrons}

\subsection{Average current}

The Coulomb interaction enhances the average current considerably above the value for non-interacting electrons, as pointed out by Ambegaokar and Eckern [13] shortly after the first persistent current experiment on many rings. Again we consider the grand potential and work out the flux-sensitive correction in a simple 


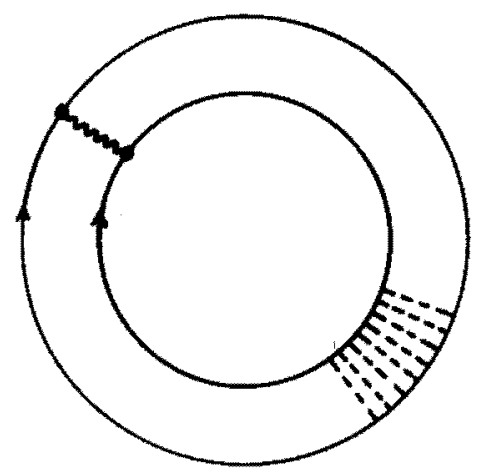

(a)

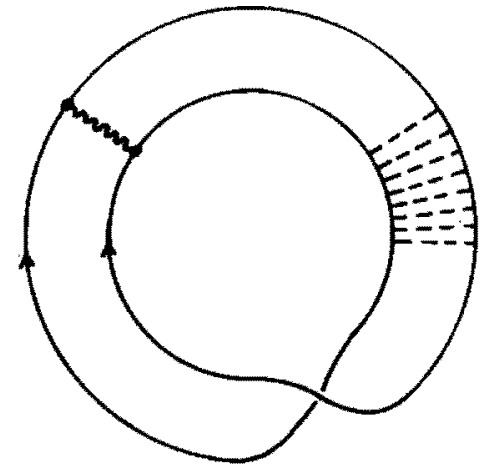

(b)

Figure 4. Interaction contributions (Hartree and Fock) to the average grand potential. The wavy line denotes the screened Coulomb interaction.

approximation (Hartree-Fock and a straightforward generalization adapted to the present problem) with respect to the interaction. As an illustration, consider the classical expression for the Coulomb interaction,

$$
\mathscr{H}_{\mathrm{int}}=\frac{1}{2} \int \mathrm{d} \mathbf{r} \int \mathrm{d} \mathbf{r}^{\prime} v\left(\mathbf{r}-\mathbf{r}^{\prime}\right) \delta n(\mathbf{r}) \delta n\left(\mathbf{r}^{\prime}\right),
$$

where $\delta n$ is the spatially varying electron density. After averaging the above with respect to the impurity configuration, we may represent the result as shown in figure $4(a)$. The corresponding exchange (Fock) contribution is given in figure $4(b)$. We have also implicitly included those terms which screen the Coulomb interaction, that is the wavy line in figure 4 (in Fourier representation) is given by

$$
v(\mathbf{k}, \omega) \approx \frac{4 \pi e^{2}}{\left(k^{2}+k_{\mathrm{TF}}^{2}\right)},
$$

where $k_{\mathrm{TF}}^{2}=8 \pi e^{2} \mathscr{N}^{0}$ is the square of the Thomas-Fermi wave-vector (i.e. the inverse screening length). Including spin summation and an extra minus for the exchange term, the final result is

$$
\left\langle\Omega_{\mathrm{int}}\right\rangle=2 \mu_{0} T \sum_{\omega>0} \sum_{\mathbf{q}} \frac{\omega}{\omega+D q^{2}} .
$$

As discussed in section 2.2 , we only need the cooperon contribution, for which

$$
\mathbf{q} \rightarrow \frac{2 \pi}{L}\left(n+\frac{2 \phi}{\phi_{0}}\right)
$$

in the above expression. We note that $\mu_{0}$, the dimensionless coupling constant, is a certain angular average of the interaction; it can be estimated to be about 0.3 for copper [13]. The final result, for zero temperature, is

$$
\left\langle I_{2 p}\right\rangle=\mu_{0} \frac{8 e E_{\mathrm{c}}}{\pi \hbar} p^{-2}
$$

The temperature dependence can very well be approximated over the relevant temperature range by an exponential, $\exp \left(-0.33 p^{2} T / E_{\mathrm{c}}\right)$, that is the scale for the 


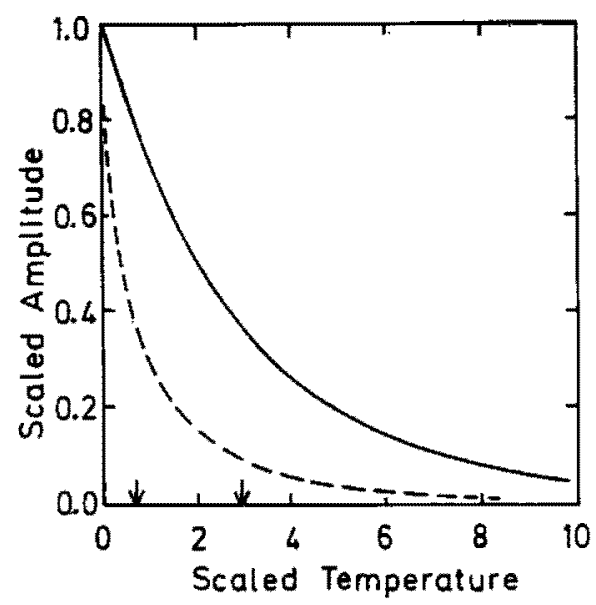

Figure 5. Scaled temperature dependence of the harmonics of the average persistent current: (-), interaction contribution; (- - ), non-interacting electrons. The arrows indicate the temperature at which the amplitudes are reduced by $e^{-1}$ respectively. The temperature scale is given by $E_{\mathrm{c}} / p^{2}$ for the $2 p$ th harmonic.

$p$ th harmonic is set by $E_{\mathrm{c}} / p^{2}$. A comparison with the temperature dependence of the non-interacting electron result, see equation (30), is given in figure 5. Note the more rapid decay of the latter with increasing temperature.

An open question, however, is the value of the coupling constant which enters equation (52). It is well known that certain higher-order terms renormalize $\mu_{0}$ logarithmically (see for example $[30,33]$ or the recent summary in $[15]$ ), such that

$$
\mu_{0} \rightarrow \mu^{*}=\frac{\mu_{0}}{1+\mu_{0} \ln \left(\epsilon_{\mathrm{F}} / E_{\mathrm{c}}\right)} .
$$

This renormalization factor is not precisely known but is most likely in the region of about $0 \cdot 1$, that is $\mu^{*} \approx 0 \cdot 1 \mu_{0}$. Possibly, the attractive electron-phonon interaction reduces it even further (and this could also lead to a different sign, implying that the system undergoes a transition to a superconducting state at extremely low temperatures). Nevertheless, we would emphasize that equation (54) (with the bare coupling constant), together with the computed temperature dependence, is in perfect agreement with the experimental findings [6]!

\subsection{Correlations}

The issue of the interaction contribution to the fluctuations of the persistent current is still open. Nevertheless we find it instructive to describe the idea behind the approach taken in [21], and to confront this idea directly with the criticism which was published immediately afterwards in [22].

It has been argued in $[21(a)]$ that the interaction breaks the symmetry of the diagram shown in figure 3 , as indicated in figure 6 by drawing a line with an asterisk. In order of magnitude, the broken line should be comparable with the line without an asterisk. As a result the logarithm has to be omitted in equation (43), and the final answer would be enhanced by a large factor, about $h / \tau E_{\mathfrak{c}}$, implying that $\left\langle I_{m}^{2}\right\rangle_{\mathrm{c}} \propto\left(e v_{\mathrm{F}} / L\right)^{2}$, roughly in agreement with experiment [7]. However, it was correctly pointed out that, if a line with an asterisk appears in one place, it should appear in all places, the symmetry would then be restored, leaving only an unimportant renormalization of the broken line, that is the scattering rate. However, 


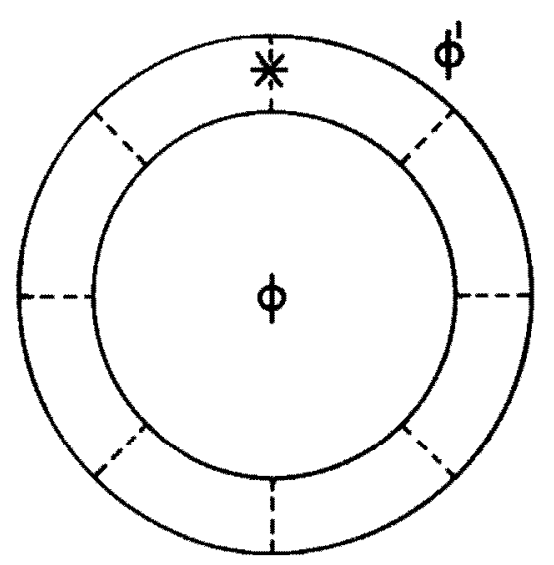

Figure 6. Contribution to the grand potential fluctuations provided that one of the impurity lines carries a different 'colour', as indicated by an asterisk. This breaks the symmetry of the diagram, greatly enhancing the final result (see section 3.2).

since the mean free path has, in any case, to be determined from (another) experiment, this brings us back to the non-interacting electron result.

In a further article $[21(b)]$, an attempt was made to explain the symmetry breaking by identifying phase-sensitive corrections to impurity lines. However, questions of double counting of diagrams remain; so this topic must be considered open for discussion.

\section{Further developments}

\subsection{Spin and magnetic field effects}

In addition to finite temperatures, there are various effects which may destroy the phase coherence around the ring and thus lead to a weakening of the persistent current, such as

(1) spin-flip scattering at (fixed) magnetic impurities,

(2) spin-orbit scattering,

(3) Zeeman splitting,

(4) penetration of the magnetic field into the metal and

(5) inelastic scattering processes.

As the latter, however, are generally small compared with the temperature effects, they will not be discussed further. In order to take spin-flip spin-orbit scattering and Zeeman splitting into account, it is necessary to determine the spin dependence of the cooperon and the diffuson [30]. In connection with the persistent currents, see, for example $[15,25]$. These effects may be important for the interpretation of the experiments; for example, it was argued (erroneously) in the first publication [6] that the sign of the observed signal, at that time believed to be diamagnetic, is due to strong spin-orbit scattering (a sign change of this type occurs for the conductivity). While it can be estimated [15] that Zeeman splitting as well as the orbital effect (field penetration) are small for the experimental situation in [6], one also finds the surprising result that the average interaction induced current is independent of the spin-orbit scattering rate. The Fock term is multiplied by $-\frac{1}{2}$ for strong spin-orbit scattering, but the Hartree contribution is multiplied by $\frac{1}{4}$, leaving the overall result unchanged. Of course, spin-flip scattering is always destructive. 
As another example, consider the persistent current fluctuations for non-interacting electrons (compare equation (43)). Owing to the symmetric appearance of cooperon and diffuson, the result is generally of the form

$$
\mathscr{M}_{\Omega}=\mathscr{F}_{C}\left(\phi+\phi^{\prime}, H+H^{\prime}\right)+\mathscr{F}_{D}\left(\phi-\phi^{\prime}, H-H^{\prime}\right)
$$

where the dependence on flux is due to equation (44), while the explicit magnetic field dependence appears because of the orbital effect and the Zeeman splitting [25]. This expression, as a generalization of equation (4), leads to the following representation:

$$
I=\sum_{m=1}^{\infty}\left[I_{m}(H) \sin \left(\frac{2 \pi \phi}{\phi_{0}}\right)+\tilde{I}_{m}(H) \cos \left(\frac{2 \pi \phi}{\phi_{0}}\right)\right] .
$$

In the absence of spin-flip scattering, $I_{m}(H)$ and $\tilde{I}_{m}(H)$ are even and odd functions respectively. Generally, they also acquire odd and even contributions (since fixed magnetic impurities break the time-reversal symmetry). For strong spin-flip scattering, for example, the cooperon is suppressed completely, while the diffusion is reduced by four [33]. As a result, the fluctuations of $I_{m}$ and $\widetilde{I}_{m}$ are equal and an eighth of the ideal value (46).

\subsection{Higher correlation functions}

There are no indications that interaction effects play a role for higher correlation functions at present. We therefore consider equation (9) for non-interacting electrons (and $k>2$ ). In view of the following differentiation with respect to $\phi_{1}, \ldots, \phi_{k}$, it is necessary to determine the contributions which have a minimal number of cooperons or diffusons (since they are small), but nevertheless a sufficient number to ensure a finite result upon differentiation. The relevant terms are shown in figure 7 for $k=3$ and $k=4$ (see also $[21,22]$ ). Details have been worked out, but we only mention

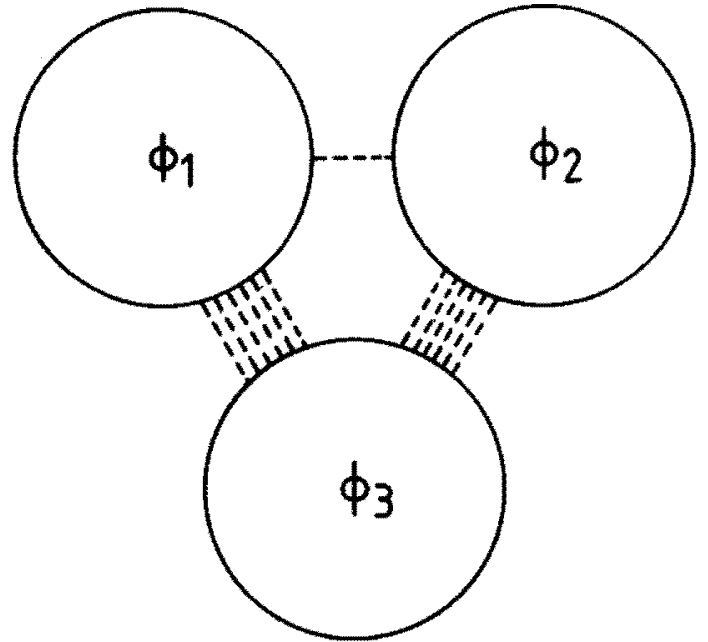

(a)

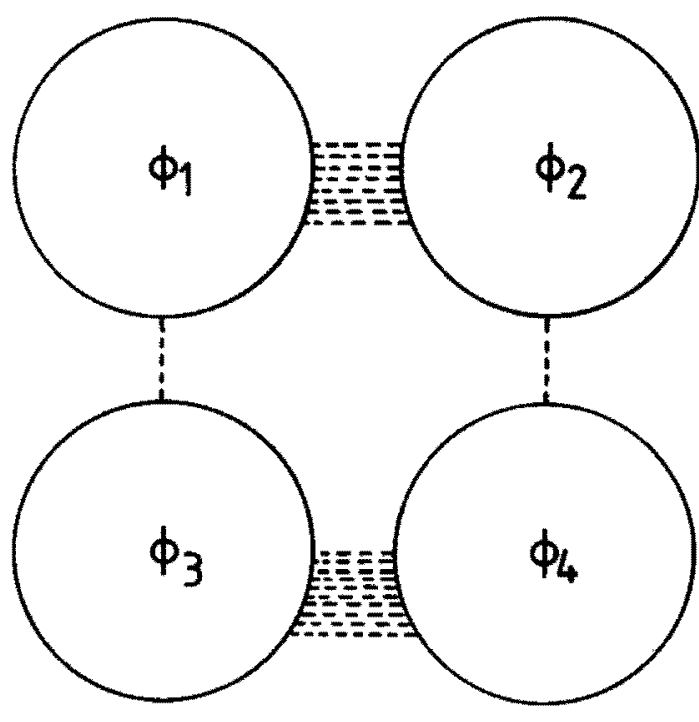

(b)

Figure 7. Dominant flux-sensitive contributions to $(a)$ the three-point and $(b)$ the four-point grand potential correlation functions. 
here that, in order of magnitude and for the flux-sensitive contribution,

$$
\mathscr{M}_{\Omega}\left(\phi_{1}, \ldots, \phi_{3}\right) \propto \frac{k_{\mathrm{F}} L}{N}\left(\frac{\hbar v_{\mathrm{F}}}{L}\right)^{3}
$$

and

$$
\mathscr{M}_{\Omega}\left(\phi_{1}, \ldots, \phi_{4}\right) \propto N^{-1}\left(\frac{\hbar v_{\mathrm{F}}}{L}\right)^{4}
$$

while higher-order terms have additional factors of $k_{\mathrm{F}} L / N \propto\left(\mathscr{A} k_{\mathrm{F}}^{2}\right)^{-1}$ and/or $N^{-1}$ and can thus be neglected for all practical purposes. Note that $\left[\mathscr{M}_{\Omega}\left(\phi_{1}, \phi_{2}, \phi_{3}\right)\right]^{1 / 3}$, depending on the experimental parameters, can be comparable with $\left[\mathscr{M}_{\Omega}\left(\phi_{1}, \phi_{2}\right)\right]^{1 / 2} \propto E_{\mathrm{c}}$ (compare equation (43)).

\subsection{Capacitance models}

In contrast with our argumentation below equation (50), if we consider a long-range interaction, it is straightforward to express the result in terms of the capacitances of the ring, according to $[27(a)]$ (see, however, also the discussion in $[27(b)]$ and the subsequent comments in $[27(c)])$

$$
\mathscr{H}_{\mathrm{int}} \rightarrow \sum_{\mathbf{k}} \frac{e^{2}}{2 C_{\mathbf{k}}} \delta N_{-\mathbf{k}} \delta N_{\mathbf{k}}
$$

For simplicity, we concentrate on $\mathbf{k}=\mathbf{0}\left(\delta N_{\mathbf{k}=\mathbf{0}} \equiv \delta N\right)$. This expression is similar to equation (29), except that now $e^{2} / 2 C$ replaces the level spacing and, since $C \approx L$ and $e^{2} \approx \hbar v_{\mathrm{F}}$ for metallic densities, we would obtain a persistent current which is comparable in size with the result for one-dimensional non-interacting electrons on a perfect ring (see section 2.1).

However, it is not possible to escape the screening phenomenon. Applying the standard random-phase approximation result,

$$
v_{0}(\mathbf{k}) \rightarrow v(\mathbf{k})=\frac{v_{0}(\mathbf{k})}{1+2 \mathscr{N}^{0} v_{0}(\mathbf{k})},
$$

we have to identify $v_{0}$ with $\left(e^{2} / C\right) \mathscr{V}$ and, considering that $\Delta \ll e^{2} / 2 C$, we finally reach the result already given in equation (29). Taking everything into consideration, this is not surprising: A strong long-ranged interaction suppresses fluctuations of the electron number, which is equivalent to considering a canonical ensemble, and thus the result is the same for both cases, and very small (since suppression of total particle number fluctuations is a global constraint [16] only).

\subsection{Superconducting rings}

The calculation of the interaction contribution to the average grand potential, and hence the persistent current, is formally identical in normal metals and in superconductors [34] above $T_{\mathfrak{c}}$, at least within the Gaussian approximation employed in $[34(a)]$. The result is

$$
\langle\Omega\rangle=T \sum_{\omega, q} \ln [\mathscr{E}(\omega, q)],
$$

where $\mathscr{E}(\omega, q)$ denote the eigenvalues of the pair propagator. Ambegaokar and Eckern [34(a)] evaluated the above expression for two cases, case $\mathrm{A}$ for $T_{\mathrm{c}} \ll T, E_{\mathrm{c}}$ and 
case $\mathrm{B}$ for $E_{\mathrm{c}} \approx T_{\mathrm{c}} \leqslant T$. In case $\mathrm{A}$, apart from the expected opposite sign, the current to a very good approximation is given by equation (52), with the dimensionless coupling constant replaced by $\approx\left[\ln \left(E_{\mathrm{c}} / T_{\mathrm{c}}\right)\right]^{-1}$. On the other hand, for case $\mathrm{B}$ and, in particular, close to the critical temperature, the classical (zero-frequency) contribution dominates, with the result

$$
\left\langle I_{2 p}\right\rangle=-\frac{8 \pi T_{\mathrm{c}}}{\phi_{0}} \exp \left(-\frac{p L}{\xi}\right),
$$

where $\xi$ is the temperature-dependent coherence length. From this expression, the average persistent current is easily determined in closed form. Note that essentially only those harmonics contribute for which $\xi>p L$, that is the coherence length must at least be comparable with the circumference. However, the divergence at small fluxes, which is a direct consequence of equation (63) for $\xi \rightarrow \infty$ (see above, equation (34) and (35)), will be removed when the fourth-order term in the Ginzburg-Landau free energy is taken into account $[34(b)]$.

Finally, we would like to discuss a situation which would be ideal for seeing both effects: 'normal' persistent currents as well as diamagnetic supercurrents [35]. Consider an experiment on a single mesoscopic superconducting ring, for which the parameters are such that $T_{\mathrm{c}} \approx E_{\mathrm{c}}$. Given the present microfabrication technology, $E_{\mathrm{c}}$ will be a few tens of millikelvins. The scale for the temperature dependence of the normal persistent current is also set by $E_{\mathrm{c}}$; so we may concentrate on the first harmonic. However, note that, in a single-ring experiment, it can be of either sign, depending on the actual impurity configuration. Furthermore, if $T_{\mathrm{c}} \sim E_{\mathrm{c}}$, the diamagnetic current induced by superconducting fluctuations is of the same order of magnitude, but of course the flux periodicity is given by $\phi_{0} / 2$. Note also that the exponent in equation (63) can also be written as $L / \xi \propto\left(T_{\mathrm{c}} / E_{\mathrm{c}}\right)^{1 / 2}$ except for the temperature dependence of the coherence length.

We would therefore suggest that, for this situation, an experiment should, at $T \approx 2 T_{\mathrm{c}}$, show both phenomena, 'normal' persistent currents (period $\phi_{0}$ ) as well as diamagnetic supercurrents (period $\phi_{0} / 2$ ), the latter of course becoming more pronounced when the critical temperature is approached. An experiment of this type would be most valuable and greatly enhance our understanding of these phenomena. Of course, if the experimental results for single rings [7] can be confirmed, the persistent current will be dominated by the 'normal' effect except for temperatures extremely close to the critical temperature.

\section{Acknowledgements}

At different stages of the investigations described in this article, one of us (U.E.) has greatly benefitted from his cooperation with Vinay Ambegaokar and Albert Schmid, as well as from many discussions with Alain Benoit and Laurent Levy.

\section{References}

[1] London, F., 1937, J. Phys., Paris, 8, 379.

[2] Hund, F., 1938, Annln Phys. (Leipzig), 32, 102.

[3] Byers, N., and YANG, C. N., Phys. Rev. Leti., 7, 46.

[4] Bloch, F., 1965, Phys. Rev, 137, A787; 1968, Ibid., 166, 415.

[5] Büttiker, Imry, Y., and Landauer, R., 1985, Phys. Lett. A, 96, 365.

[6] Levy, L. P., Dolan, G., Dunsmur, J., and Bouchiat, H., 1990, Phys. Rev. Lett., 64, 2074; Levy, L. P., 1990, Physica B, 167, 50. 
[7] Chandrasekhar, V., Webb, R. A., Brady, M. J., Ketchen, M. B., Gallagher, W. J., and KleinSASSER, A., 1991, Phys. Rev. Lett., 67, 3578.

[8] (a) Mailly, D., Chapelier, C., and Benoit, A., 1993, Phys. Rev. Lett., 70, 2020; (b) Reulet, B., Ramin, M., Bouchiat, H., and Mailly, D., 1995, Phys. Rev. Lett., 75, 124.

[9] Cheung, H.-F., Gefen, Y., and Riedel, E. K., 1988, IBM $J$ Res. Dev., 32, 359; Zoller, H., 1990, Diploma Thesis, University of Karlsruhe.

[10] Entin-Wohlman, O., and Gefen, Y., 1989, Europhys. Lett., 8, 477.

[11] Cheung, H. F., Riedel, E. K., and Gefen, Y., 1989, Phys. Rev. Lett., 62, 587.

[12] Bouchiat, H., and Montambaux, G., 1989, J. Phys., Paris, 50, 2695; Montambaux, G., Bouchiat, H., Sigeti, D., and Friesner, R., 1990, Phys. Rev. B, 42, 7647.

[13] Ambegaokar, V., and Eckern, U., 1990, Phys. Rev. Lett., 65, 381; 1991, Ibid., 67, 3192 (comment).

[14] ImRY, Y., 1991, Quantum Coherence in Mesoscopic Systems, Advanced Study Institute Series, Series B: Physics, Vol. 254, edited by B. Kramer (New York: Plenum), p. 221.

[15] ECKERN, U., 1991, Z. Phys. B, 82, 393.

[16] Schmid, A., 1991, Phys. Rev. Lett., 66, 80.

[17] vON OPPEN, F., and RIEDEL, E. K., 1991, Phys. Rev. Lett., 66, 84.

[18] Altshuler, B. L., Gefen, Y., and Imry, Y., 1991, Phys. Rev. Lett., 66, 88.

[19] Efetov, K. B., 1991, Phys. Rev. Lett., 66, 2794.

[20] Altland, A., Iida, S., Müller-Groeling, A., and Weidenmüller, H. A., 1992, Europhys. Lett., 20, 155; 1992, Ann. Phys. (N.Y.), 219, 148.

[21] (a) ECKERN, U., and ScHMD, A., 1992, Europhys. Lett., 18, 457; (b) 1993, Annln. Phys. (Leipzig), 2, 180.

[22] Smitt, R. A., and Ambegaokar, V., 1992, Europhys. Lett., 20, 161.

[23] Riedel, E. K., and von OpPEn, F., 1993, Phys. Rev. B, 47, 15449.

[24] Loss, D., 1992, Phys. Rev. Lett., 69, 343.

[25] ECKern, U., 1993, Phys. Scri. T, 49, 338.

[26] Müller-Groeling, A., WeidenMứller, H. A., and LewenkopF, C. H., 1993, Europhys. Lett., 22, 193; WidenMüller, H. A., 1993, Physica A, 200, 104.

[27] (a) Kopietz, P., 1993, Phys. Rev. Lett., 70, 3123; (b) Altshuler, B. L., 1992, Nanostructures and Mesoscopic Phenomena, edited by W. P. Kirk and M. A. Reed (San Diego, California: Academic Press), p. 405; (c) Vignale, G., 1994, Phys. Rev. Lett., 72, 433, and Altland, A., and Gefen, Y., 1994, Phys. Rev. Lett., 72, 2973.

[28] Abraham, M., and Berkovits, R., 1993, Phys. Rev. Lett., 70, 1509.

[29] Washburn, S., and WebB, R. A., 1986, Adv. Phys., 35, 375.

[30] Altshuler, B. L., Aronov, A. G., Khmelnitskit, D. E., and Larkin, A. I., 1980, Quantum Theory of Solids, edited by I. M. Lifshitz (Moscow: Mir), p. 130; ALtsHuler, B. L., and ArNov, A. G., 1985, Electron-Electron Interactions in Disordered Systems, edited by A. L. Efros and M. Pollak (Amsterdam: North-Holland), p. 1.

[31] Abrikosov, A. A., Gorkov, L. P., and Dzyaloshinskir, I. Ye., 1965, Quantum Field Theoretical Methods in Statistical Physics (Oxford: Pergamon).

[32] Efetov, K. B., 1983, Adv. Phys., 32, 53; Verbaarschot, J. J. M., Weidenmüller, H. A., and Zirnbauter, M. R., 1985, Phys. Rev., 129, 367.

[33] Altshuler, B. L., and ShklovskiI, B. I., 1987, Soviet Phys. JETP, 64, 127.

[34] (a) Ambegaokar, V., and Eckern, U., 1990, Europhys. Lett., 13, 733; 1991, Phys. Kev. B, 44, 10358; (b) VON OPPEN, F., 1993, Ph.D. Thesis, University of Washington, chapter 8 .

[35] ECKERN, U., 1994, Physica B, 203, 448. 